\title{
The Ideal and the Real
}

In this chapter we first attempt to persuade (or remind) the reader that much healthcare departs from the care envisaged by standards and guidelines. We appreciate that standards and guidelines need considerable interpretation and adaptation for patients with multiple conditions (Tinetti et al. 2004) and that even the simplest conditions require consideration of personal preferences and other factors. However we are concerned primarily with the basic fact that the care provided to patients often does not reach the standard that professionals intend to deliver and which professional consensus would regard as reasonable and achievable. Clinical processes and systems are often unreliable and in fact many patients are harmed by the healthcare intended to help them. All this is to some degree obvious to anyone who works at the frontline or studies healthcare deeply. One of the questions we address in this book is how to manage the gap between the 'real and the ideal' and how best to manage the risks to patients.

Many factors conspire to make optimal care both difficult to define and difficult to achieve (Box 2.1). The vulnerabilities of the system, personal attitudes, team dynamics and a variety of external pressures and restraints combine to produce a 'migration' away from best practice. This in turn means that clinical staff are engaged in constant adaptation, detecting problems and responding to them. Safety is in a very real sense achieved by frontline practitioners rather than imposed by standards. We will develop this further in later chapters to argue that safety strategies to manage these risks need to foster these adaptive capacities both at an individual and organisational level. 


\section{Box 2.1 Observation of Patients at Risk of Suicide: When Working Conditions} Make It Difficult to Follow Procedures

Over a 1 year period there were on average 18 suicides by in-patients under observation per year in hospitals in the United Kingdom. Ninety-one percent of deaths occurred when patients were under level 2 (intermittent) observation.

Deaths under observation tended to occur when policies or procedures (including times between observations) were not followed, for example:

- When staff are distracted by other events on the ward

- At busy periods, such as between 7.00 and 9.00

- When there are staff shortages

- When ward design impedes observation.

National Confidential Inquiry into Suicide and Homicide by People with Mental Illness (2015)

\section{The Day-to-Day Realities of Healthcare}

When we are working we are usually preoccupied with the task in hand and do not have the attentional capacity to simultaneously reflect on the working environment or remember all the difficulties encountered during the day. Furthermore, we are not easily able to aggregate our experience over long time periods. For instance, a doctor may know that notes are often missing in the clinic but will struggle to estimate how often this happens over a year. In addition it is very difficult for individuals to gain a true understanding of the failures and vulnerabilities across an entire technical area. Patients and families have a privileged view in that they alone follow the full story of care but our view as patients is obviously partial in that we cannot know the wider workings of the hospital or clinic. All these factors combine to make it difficult for any individual to monitor or assess the overall standard of care. There is however ample evidence to support the simple idea that care often falls below the standard expected. Let us consider some examples.

\section{Comparing Actual Care with the Care Intended by Guidelines}

Major studies in both the United States and Australia suggest that patients typically receive only a proportion of the care indicated by guidelines. Studies in the United States suggest that many patients received only about half of recommended care, though other patients receive investigations and treatment that are unnecessary (McGlynn et al. 2003). In a major recent Australian study adult patients received only $57 \%$ of recommended care with compliance ranging from $13 \%$ for alcohol dependence to $90 \%$ for coronary artery disease (Runciman et al. 2012). These studies did not assess the direct impact on the patients concerned, but other studies have linked failures in the care provided with subsequent harm. For instance Taylor and colleagues (2008) 
interviewed 228 patients during and after their treatment and found 183 service quality deficiencies, each of which more than doubled the risk of any adverse event or close call for that patient. Service quality deficiencies involving poor coordination of care were particularly associated with the occurrence of adverse events and medical errors. In another example physicians reviewed 1566 case notes from 20 English Hospitals writing judgment-based comments on the phases of care provided and on care overall. About a fifth of the patients were considered to have received less than satisfactory care, often experiencing a series of adverse events (Hutchinson et al. 2013).

\section{Reliability of Clinical Systems in the British NHS}

Some healthcare processes, such as the administration of radiotherapy, achieve very high levels of reliability. Other processes are haphazard to say the least. Burnett and colleagues (2012) examined the reliability of four clinical systems in the NHS: clinical information in surgical outpatient clinics, prescribing for hospital inpatients, equipment in theatres, and insertion of peripheral intravenous lines. Reliability was defined as $100 \%$ fault free operation when, for example, every patient had the required information available at the time of their appointment.

Reliability was found to be between 81 and $87 \%$ for the systems studied, with significant variation between organisations for some systems; the clinical systems therefore failed on 13-19\% of occasions. This implies, if these findings are typical, that in an English hospital: doctors are coping with missing clinical information in three of every 20 outpatient appointments and there is missing or faulty equipment in one of seven operations performed. In each case where measured, about $20 \%$ of reliability failures were associated with a potential risk of harm. On this basis it is hardly surprising that patient safety is routinely compromised in NHS hospitals and that clinical staff come to accept poor reliability as part of everyday life.

\section{Following the Rules: Reliability of Human Behaviour}

Delivering safe, high quality care is an interplay between disciplined, regulated behaviour and necessary adaptation and flexibility. Rules and procedures are never a complete solution to safety and sometimes it is necessary to depart from standard procedures in the pursuit of safety. However, protocols for routine tasks are standardised and specified precisely because those tasks are essential to safe, high quality care.

Protocols of this kind are equivalent to the safety rules of other industries defined ways of behaving when carrying out safety-critical tasks (Hale and Swuste 1998). Examples in healthcare include: checking equipment, washing your hands, not prescribing dangerous drugs when you are not authorised to, following the procedures when giving intravenous drugs and routinely checking the identity of a patient. Such standard routines and procedures are the bedrock of a safe organisation, but there is ample evidence that such rules are routinely ignored:

- Hand washing. Contamination through hand contact is a major source and hand hygiene a major weapon in the fight against infection (Burke 2003). Studies have 
found that average levels of compliance, before major campaigns were instituted, have varied from 16 to $81 \%$ (Pittet et al. 2004).

- Intravenous drug administration. Studies have found that over half involve an error, either in the preparation of the drug or its administration. Typical errors were preparing the wrong dose or selecting the wrong solvent (Taxis and Barber 2003).

- Prophylaxis against infection and embolism. Only $55 \%$ of surgical patients receive antimicrobial prophylaxis (Bratzler et al. 2005) and only $58 \%$ of those at risk of venous thromboembolism receive the recommended preventive treatment (Cohen et al. 2008).

The causes of departure from standards are many. In some settings the working environment is reasonably calm and orderly so staff are able to follow clear protocols and abide by core standards. In other settings however the pressures are great, the environment noisy and chaotic and staff are essentially just trying to do the best they can in the circumstances. In any systems there are pressures for greater productivity, less use of resources and occasions where missing or broken equipment forces adaptations and short cuts; add to this that we all, occasionally or frequently, are in a rush to get home, get on to the next case, tired or stressed and apt to cut corners. Standards may be unrealistic or too complex; staff may not be sufficiently skilled or have not received the necessary training. Working in such conditions is an everyday occurrence for many clinicians and acts as a constant reminder of the care they would like to give and the reality of the care they are able to provide. Over time however these departures from standards can become increasingly tolerated and eventually invisible (Box 2.2).

Box 2.2 External Pressures and Gradual Migration to the Boundary of Safety

Occasional lapses can become more tolerated over time and systems can become gradually more degraded and eventually dangerous. The phrase 'illegal normal' captures the day-to-day reality of many systems in which deviations from standard procedures (the illegal) are widespread but occasion no particular alarm (they become normal). The concept of routine violations is not part of the thinking of managers and regulators; in truth it is a very uncomfortable realisation that much of the time systems, whether healthcare, transport or industry, operate in an 'illegal-normal' zone. The system continues in this state because the violations have considerable benefits, both for the individuals concerned and for managers who may tolerate them, or even encourage them, in the drive to meet productivity standards.

Over time these violations can become more frequent and more severe so that the whole system 'migrates' to the boundaries of safety. Violations are now routine and so common as to be almost invisible to both workers and managers. The organisation has now become accustomed to operating at the margins of safety. At this stage, any further deviance may easily result in patient harm, and would generally be considered as negligent or reckless conduct (Amalberti et al. 2006). 


\section{The Ideal and the Real: Five Levels of Care}

We now consider the implications of the gap between the care envisaged by standards and guidelines and the care actually given to patients. We have found it useful to distinguish five levels of care each departing further from the ideal and, we suggest, increasing probability of harm as one moves down the levels.

1. Level 1 corresponds to optimal care envisaged by standards (though truly optimal care can never be encapsulated in standards). These standards are set out by national and professional organisations and represent a consensus on what can be regarded as the optimum care achievable within current cost constraints. This level provides a shared ideal reference of excellent care, although it is seldom fully achieved across an entire patient journey.

2. Level 2 represents a standard of care which experts would judge as both providing a good outcome for the patient and also achievable in day to day practice. The care is of good standard and the outcome is good, even though there may be minor variations and problems. Any departures from best practice are relatively unimportant in the overall care provide to the patient.

3. Level 3 represents the first level where the safety of the patient may be compromised. We consider, for reasons given above, that a considerable amount of the healthcare that patients receive falls broadly into this category. At this level there are frequent departures from best practice which occur for a wide variety of different reasons and are a potential threat to patients. There may for example not be a timely monitoring of anticoagulation level after prescription of heparin. This level has been previously described as the 'illegal normal' (Amalberti et al. 2006) (Box 2.2).

4. Level 4 represents a departure from standards which is sufficient to produce avoidable harm. For example, a 68 year old patient undergoes a cholecystectomy and contracts a urinary catheter infection after surgery. Analysis of the event showed that the catheter was not checked regularly and was left in place too long. This was a clear departure from expected care. However treatment was rapidly instituted and the infection was under full control after 10 days. The patient suffered avoidable harm and had to stay in hospital an additional week but then recovered completely.

5. Level 5 refers to care that is poor over a longer period and places the patient at risk of substantial and enduring harm. For instance if, in the case described above, the patient not only contracted the infection but it was then not recognised and not treated effectively. This would result in at best a very prolonged recovery and increased frailty but also a potentially fatal outcome.

Broadly speaking we see Level 1 as optimal care, certainly a valuable aspiration and inspiration but very difficult to achieve in practice and in many settings not easy to define. Optimal care is relatively easy to specify in a highly standardised and structured clinical setting but in many environments, particularly primary care, the care provided necessarily evolves and unfolds in a complex social context (Box 2.3). Level 2 is a more realistic level of care where there are minor imperfections but 
clinical care is of a very good standard. Level 3 is a distinct deterioration with multiple lapses of care but not sufficient to greatly affect long term outcome. Levels 4 and 5 in contrast offer potential for harm, either through omission of critical aspects of care, serious errors or neglect. In the common understanding of these terms the ambitions of high quality care are associated with Levels 1 and 2, and those concerned with safety aiming to avoid levels 4 and 5 .

\section{Box 2.3 Optimal Care Can Often Not Be Precisely Defined}

There are many clinical situations in which optimal care cannot be precisely defined. This may be because the disease is not well understood, is rare or expressed in an unusual manner. More commonly though the patient, often frail or elderly, is suffering from a number of different conditions presenting a complicated and changing picture. In these cases, common in primary care and mental health, clinical judgement and sensitive shared decisions are to the fore:

I don't believe that in much of what we do in healthcare, particularly in primary care, we can define what we mean by 'excellence', nor can we codify it though guidelines and standards. We can reduce health provision to its component parts and pretend that these reflect the whole but this ignores the inherent paradoxes of competing goods and trade-offs with other objectives. The thinking that dominates the safety world is sometimes too rational. What I see is lots of thoughtful clinicians who understand the discrepancy between the ideal and the real, for whom the tension is always on their agenda and who thoughtfully manage these tensions because they accept that they live in a world that wants to simplify (M Marshall, 2015, personal communication)

Figure 2.1 is similar to many diagrams which represent variation in standards of care and which distinguish good, average and poor units or organisations. Certainly some organisations deliver poor care for sustained periods of time and even national services can have periods of high risk of harm at times of crisis. However we intend to capture a more fluid reality in which any patient is at risk of a sudden decline in standards, and at risk of harm, on many occasions during their healthcare journey. Safety can be eroded quite suddenly in any team or organisation, just as there is always some risk of accident with the safest car driven by the best driver on the safest road. We are therefore not only concerned with strategies which may support struggling teams or organisations but also with developing strategies and interventions to manage risk on a day-to-day basis.

\section{The Cumulative Impact of Poor Quality Care}

Patients can receive some treatment of poor quality, in the sense of haphazard and patchy adherence to accepted standards, and still not come to any harm. We suggest, however, harm is much more probable when healthcare moves further from best 


\section{5 levels of care}

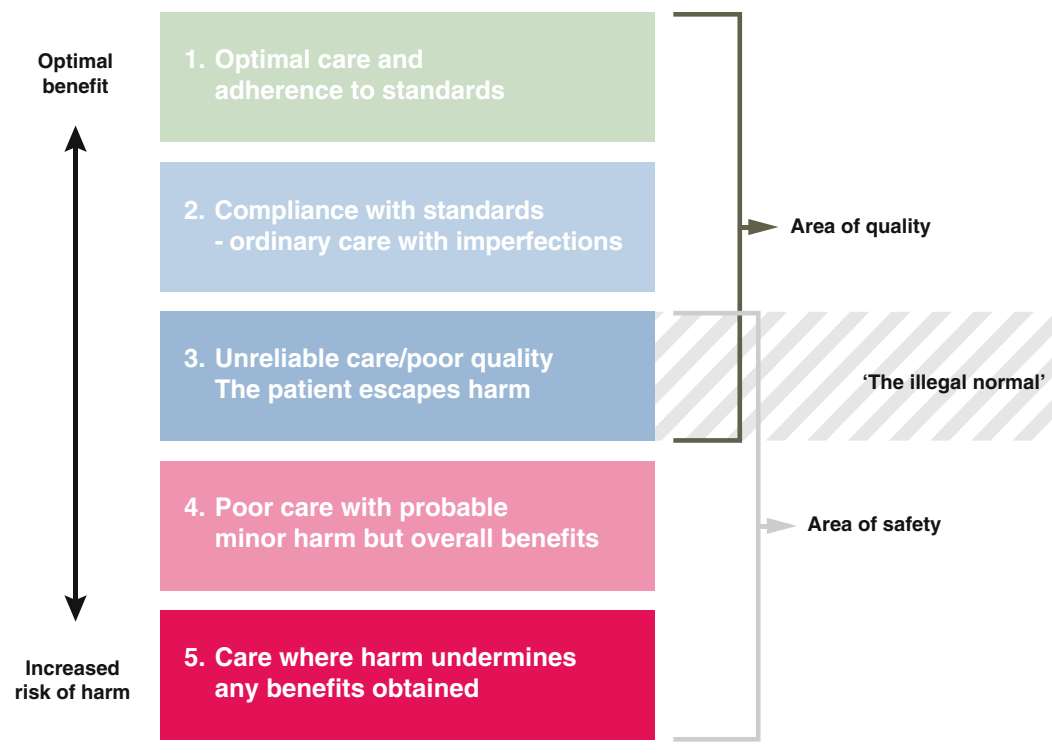

Fig. 2.1 Five levels of care

practice (Levels 4 and 5). This is partly because obvious lapses in standards (such as not checking patient identity) may lead to immediate harm but the greater danger to patients probably comes from the cumulative impact of minor problems (Hutchinson et al. 2013).

Suppose for example, a fit and well 26 year old patient has planned abdominal surgery for inflammatory bowel disease on a Wednesday. Due to a shortage of beds, the patient is placed on the orthopaedic ward, with nurses, pharmacists and other clinicians who are not used to looking after this type of problem. The operation is difficult and complex; a piece of bowel was removed and a new join made between the remaining ends of the bowel. On Saturday evening the patient has an episode of fever at $39{ }^{\circ} \mathrm{C}$ and abdominal pain, which could indicate that the new join is leaking. The young doctor on duty over the weekend is a locum, who does not know the patient. She tries to read the operation note but it is not completely legible. She does not appreciate the potential seriousness and does not seek more senior advice. Over the next $24 \mathrm{~h}$ the patient continues to deteriorate but the staff do not appreciate the significance of the symptoms. By Monday morning the patient is so unwell that he suffers a cardiac arrest and eventually dies on Monday evening after a futile return to the operating theatre during the day.

This scenario describes a series of relatively ordinary and commonplace lapses, omissions and events which together have a catastrophic effect. Clearly there were 
problems in assessment of symptoms, escalation, record keeping, communication, coordination of care and management of bed availability, possibly exacerbated by external pressures. None of the individual lapses and problems is out of the ordinary or particularly shocking - but they combine to create catastrophe.

The impact of the cumulative effects of poor care suggests that we may need to widen the time frame of analyses of adverse events and poor outcomes. This will be especially important once we consider safety in the home and community and in the context of the overall impact of healthcare on a person's life and well-being. However we may also see a much greater incidence of harm due to cumulative minor failure in the future owing to the number and complexity of transitions along the patient journey.

Consideration of the cumulative effects of poor care also has implications for how we assess priorities for patient safety. Dramatic incidents, such as deaths from spinal injection of vincristine (Franklin et al. 2014), attract considerable attention and are tragic for the people involved but they tend to skew the direction of patient safety initiatives towards comparatively rare events. In surgery the cases that attract most attention are those with sudden, dramatic outcomes with fairly immediate causes. These are incidents such as operating on the wrong patient or retained foreign body which are rare but frequently disastrous when they do occur; they are low risk but 'high dread' in the language of the psychology of risk. However surgical patients run much greater risks from care that is simply of poor standard for whatever reason. There is for instance a huge variation in mortality from surgery across Europe. In a major recent review 46,539 patients were studied of whom 1855 (4\%) died before hospital discharge (Pearse et al. 2012). Crude mortality rates varied widely between countries range from $1.2 \%$ for Iceland to $21.5 \%$ for Latvia. Substantial differences remained even after adjustment for confounding variables. This suggests that much of the care provided is, in our terms, of levels 3, 4 and 5 even by the standards of individual countries. Once we begin to see safety in these terms it is clear that the harm from poor management of post-operative complications dwarfs the much more prominent problem of surgical never events.

We need therefore to reflect on the broader priorities from a population health perspective. This process has already begun with the increased attention given to programmes to reduce falls, pressure ulcers, acute kidney injury and infections of all kinds. The scope of patient safety needs to further expand to embrace consideration of poor care of all kinds and to integrate with those seeking to understand and reduce the sources of variability. We should also remember that most studies at the hospital level focus on one particular type of adverse event (such as hospital acquired infection) or one service (such as surgery). Very few studies assess the whole spectrum of incidents afflicting patients or assess their cumulative impact over time. 


\section{Explicit Discussion of the Real Standard of Care Is Critical}

We now come to a central problem and challenge which is that the standard of usual care cannot easily be explicitly discussed. It is of course understood implicitly within clinical teams and each new member is socialised into accepting the standards of care in that particular environment which may be either higher, or lower, than they are used to. When people join a unit, or spend time in another unit, there may be a sudden shock of recognition of a very different standard and tolerance for departures from standards. There is huge variation in different clinical teams in what they regard as good enough care which is influenced by the social norms and values of that particular setting. Care that is viewed as ordinary on one ward might be seen as being a major lapse in standards on another. Most clinicians are aware that much care is in the 'illegal normal' range and immediately recognise this concept when it is presented. They know that the care they provide often falls short of the care they would like to provide but they are adept at navigating the healthcare system to provide the best care they can in the circumstances.

Organisations however, and still more governments, cannot easily openly say that much care is at level 3 and routinely dips to levels 4 and 5. This has some important consequences for the management of risk. First, it becomes very difficult to study or to value the many adaptive ways in which staff cope in difficult environment to prevent harm coming to patients. Second, and most important to our arguments, attempts to improve safety may not be targeting the right levels or the right behaviours. We will argue later that most safety interventions are essentially attempts to improve reliability and, ultimately, to move all care towards Level 1. This is an important and necessary strategy but, in our view, only applicable in some circumstances. This approach need to be supplemented by strategies that aim to maintain care at Level 3 and prevent decay into levels 4 and 5. We might express this by saying that in the day to day provision of care it is more urgent that our systems prioritise achieving reliable basic standards than striving for unachievable ideals. If care is generally at Level 3 then the principal aim might be to improve reliability and move to Level 2 . If however care is often at level 4 or 5, that is frankly dangerous, then the detection and response to potential dangers might be a higher priority (Fig. 2.2).

The aspirations to excellence are important and should not be mocked or derided as unrealistic. The problem is that the rhetoric of excellence masks the urgently needed discussion of the realities of 'usual care'. If our aspiration becomes only to deliver 'good enough' care then there is a danger of increased variation, declining standards and increased hazard. The definition and aspiration of optimum quality remains critical - but so does an explicit discussion of the current reality. 


\section{What Is the Impact of Improving Quality Standards?}

Innovation and the aspiration to continually improve are at the heart of medicine. However the introduction of new treatments or new standards of care may place unrealistic demands on both staff and organisations. Stroke for example was at one time regarded as untreatable. Brain cells were thought to die within minutes after a stroke began, and medical treatment largely consisted of caring for the patient and "wait and see". We have known for a decade now that treatment following a stroke, especially if begun within $3 \mathrm{~h}$ of onset, can preserve brain tissue. Guidelines typically recommend a door-to-needle time of $60 \mathrm{~min}$. However in 2011 only one third of American patients were treated within the guideline-recommended door-toneedle times (Fonarow et al. 2013). Many countries have instituted major programmes to improve the efficiency of treatment for stroke which have led to great improvements in outcome.

\section{5 levels of care}

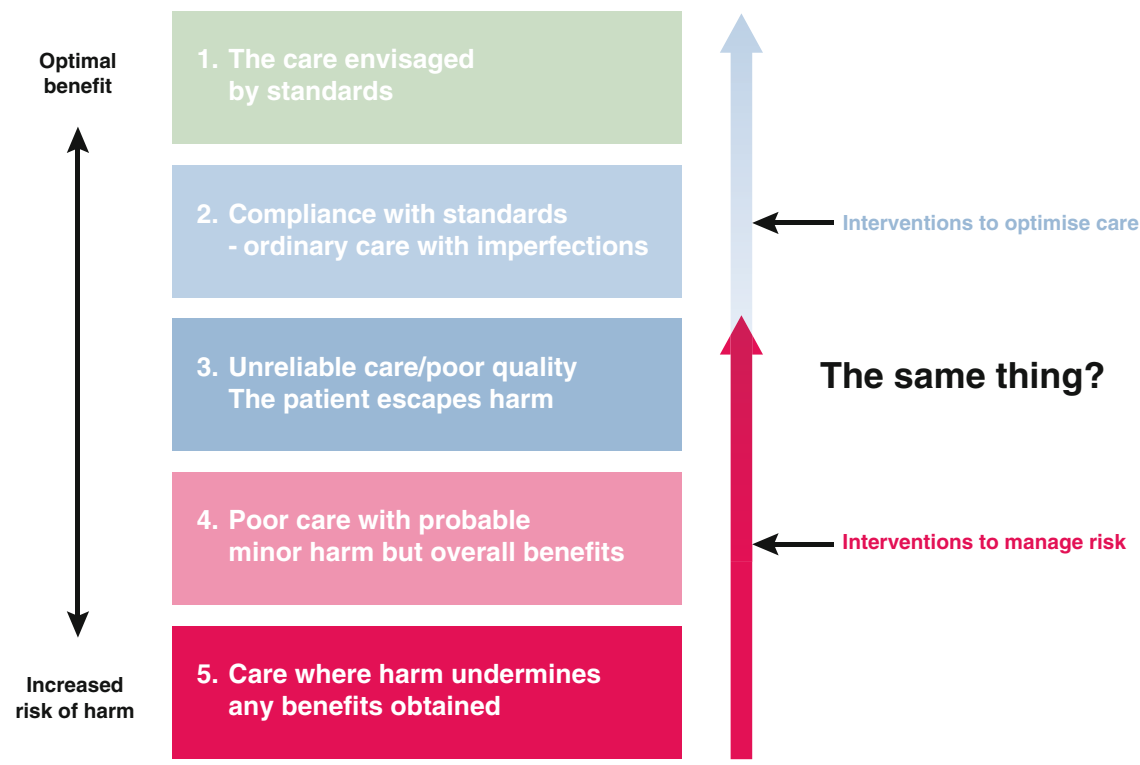

Fig. 2.2 Optimisation and risk management 
We are not criticising the care given or the delays in bringing in new standards of delivery. Rather we are pointing to the inevitable increase in departures from guidelines which result when a new standard is specified. Ten years ago there would have been few 'incidents' relating to failures in the early treatment of stroke because standards of care had not been introduced. We can now, because of improved care, point to numerous serious incidents because many patients cannot access to care within the $3 \mathrm{~h}$ of onset due to a failures in the healthcare system. As standards improve we are therefore likely to have an increasing number of incidents which are concerned with omissions of care. What counts as an 'incident' in 2015 may simply have been ordinary practice in 2005 ; this is a very common consideration in legal cases which are being decided some years after the initial event. The new standards, hugely beneficial for patients, create new kinds of incidents and safety problems (Vincent and Amalberti 2015).

\section{Levels of Care and Strategies for Safety Improvement}

Improving the standard of care delivered and the gradual setting of higher standards is of course a positive and necessary aspiration. However when doing this we need to recognise that we are redefining both quality and safety and increasing the pressure on individuals and organisations. Champions of the new standards will emerge and bring about change but many organisations will take time to meet the new standard and weaker organisations may even be destabilised because of the increasing demand.

We have at present very few strategies for managing the transitional period or for responding constructively to the inevitable gap between expected standards and organisational reality. During these transition periods we will need to do more than simply exhort and harass organisations to meet the new standards. We must also recognise the inevitable lag and employ strategies that emphasise the detection of problems, awareness of conditions which degrade safety and individual and enhance team based management of potentially harmful care. These arguments will be fully developed in Chap. 7. 


\section{Key Points}

- Many patients do not receive the care intended. We can do a great deal to increase reliability and achieve higher standards of care. However we believe that in healthcare there will always be a gap between the ideal and the real.

- We distinguish five levels of care each departing further from the ideal and, we suggest, increasing probability of harm as one move down the levels.

- Level 1 corresponds to the optimum care envisaged by standards. This level provides a shared reference of excellent care, although it is seldom fully achieved across an entire patient journey.

- Level 2 represents a standard of care which experts would judge as both providing a good outcome for the patient and also achievable in day to day practice

- Level 3 represents the first level in which the safety of the patient is threatened. At this level there are frequent departures from best practice which occur for a wide variety of different reasons.

- Level 4 represents a departure from standards which is sufficient to produce avoidable harm but not sufficient to substantially affect the overall outcome.

- Level 5 refers to care that is poor over a longer period and places the patient at risk of substantial and enduring harm.

- We suggest that organisations and government find it difficult to openly discuss the daily threats and variations in standards of care. This has important consequences. First, it becomes very difficult to study or value the many ways staff adapt to prevent harm coming to patients. Second, attempts to improve safety may not be targeting the right levels or the right behaviours.

- The aspirations to excellence are important and should not be derided as unrealistic. The problem is that the rhetoric of excellence masks the urgently needed explicit discussion of the realities of usual care which is a critical first step in the effective management of risk.

- We propose that most safety interventions are essentially attempts to improve reliability. This is an important and necessary approach but needs to be complemented by additional strategies that aim to manage risk and protect patients from serious failures in care.

Open Access This chapter is distributed under the terms of the Creative Commons Attribution Noncommercial License, which permits any noncommercial use, distribution, and reproduction in any medium, provided the original author(s) and source are credited. 


\section{References}

Amalberti R, Vincent C, Auroy Y, de Saint Maurice G (2006) Violations and migrations in health care: a framework for understanding and management. Qual Saf Health Care 15(suppl 1):i66-i71

Bratzler DW, Houck PM, Richards C, Steele L, Dellinger EP, Fry DE, Red L (2005) Use of antimicrobial prophylaxis for major surgery: baseline results from the National Surgical Infection Prevention Project. Arch Surg 140(2):174-182

Burke JP (2003) Infection control-a problem for patient safety. New England Journal of Medicine, 348(7):651-656

Burnett S, Franklin BD, Moorthy K, Cooke MW, Vincent C (2012) How reliable are clinical systems in the UK NHS? A study of seven NHS organisations. BMJ Qual Saf. doi:10.1136/ bmjqs-2011-000442

Cohen AT, Tapson VF, Bergmann JF, Goldhaber SZ, Kakkar AK, Deslandes B, Huang W, Zayaruzny M, Emery L, Anderson FA Jr, Endorse Investigators (2008) Venous thromboembolism risk and prophylaxis in the acute hospital care setting (ENDORSE study): a multinational cross-sectional study. Lancet 371(9610):387-394

Fonarow GC, Liang L, Smith EE, Reeves MJ, Saver JL, Xian Y et al (2013) Comparison of performance achievement award recognition with primary stroke centre certification for acute ischemic stroke care. J Am Heart Assoc 2(5), e00045

Franklin BD, Panesar S, Vincent C, Donaldson L (2014) Identifying systems failures in the pathway to a catastrophic event: an analysis of national incident report data relating to vinca alkaloids. BMJ Qual Saf 23(9):765-772

Hale AR, Swuste PHJJ (1998) Safety rules: procedural freedom or action constraint? Saf Sci 29(3):163-177

Hutchinson A, Coster JE, Cooper KL, Pearson M, McIntosh A, Bath PA (2013) A structured judgement method to enhance mortality case note review: development and evaluation. BMJ Qual Saf 22(12):1032-1040

McGlynn EA, Asch SM, Adams J, Keesey J, Hicks J, DeCristofaro A, Kerr EA (2003) The quality of health care delivered to adults in the United States. N Engl J Med 348(26):2635-2645

National Confidential Inquiry into Suicide and Homicide by People with Mental Illness (NCISH) (2015) In-patient suicide under observation. University of Manchester, Manchester

Pearse RM, Moreno RP, Bauer P, Pelosi P, Metnitz P, Spies C, Rhodes A (2012) Mortality after surgery in Europe: a 7 day cohort study. Lancet 380(9847):1059-1065

Pittet D, Simon A, Hugonnet S, Pessoa-Silva CL, Sauvan V, Perneger TV (2004) Hand hygiene among physicians: performance, beliefs, and perceptions. Ann Intern Med 141(1):1-8

Runciman WB, Hunt TD, Hannaford NA, Hibbert PD, Westbrook JI, Coiera EW, Braithwaite J (2012) CareTrack: assessing the appropriateness of health care delivery in Australia. Med J Aust 197(10):549

Taxis K, Barber N (2003) Causes of intravenous medication errors: an ethnographic study. Qual Saf Health Care 12(5):343-347

Taylor BB, Marcantonio ER, Pagovich O, Carbo A, Bergmann M, Davis RB, Weingart SN (2008) Do medical inpatients who report poor service quality experience more adverse events and medical errors? Med Care 46(2):224-228

Tinetti ME, Bogardus ST Jr, Agostini JV (2004) Potential pitfalls of disease-specific guidelines for patients with multiple conditions. N Engl J Med 351(27):2870-2874

Vincent C, Amalberti R (2015) Safety in healthcare is a moving target. BMJ Qual Saf. doi:10.1136/ bmjqs-2015-004403 